Malgré sa grande importance pratique, la théorie des problèmes inverses reste mal connue. Et pourtant elle est presque omniprésente : avec le GPS, du trajet automobile en ville à la mesure de la vitesse des neutrinos, avec la sismologie,

la prospection pétrolière, la découverte de nouvelles planètes, I'imagerie médicale, la reconnaissance des caractères, la cristallographie...

La physique s'est construite par l'élaboration de théories, c'est-à-dire par la résolution de problèmes directs, où l'on passe d'un modèle aux données mesurables correspondantes.

Le passage des données aux paramètres du modèle la résolution d'un problème dit « inverse »- est une démarche en quelque sorte opposée à la démarche naturelle de la physique. Mais elle est essentielle dans toutes les disciplines où celle-ci intervient.

\title{
Des problèmes inverses en physique
}

Georges Jobert (jobert.g@wanadoo.fr)

Professeur honoraire à I'Université Pierre et Marie Curie - Ancien directeur de l'Institut de Physique du Globe de Paris

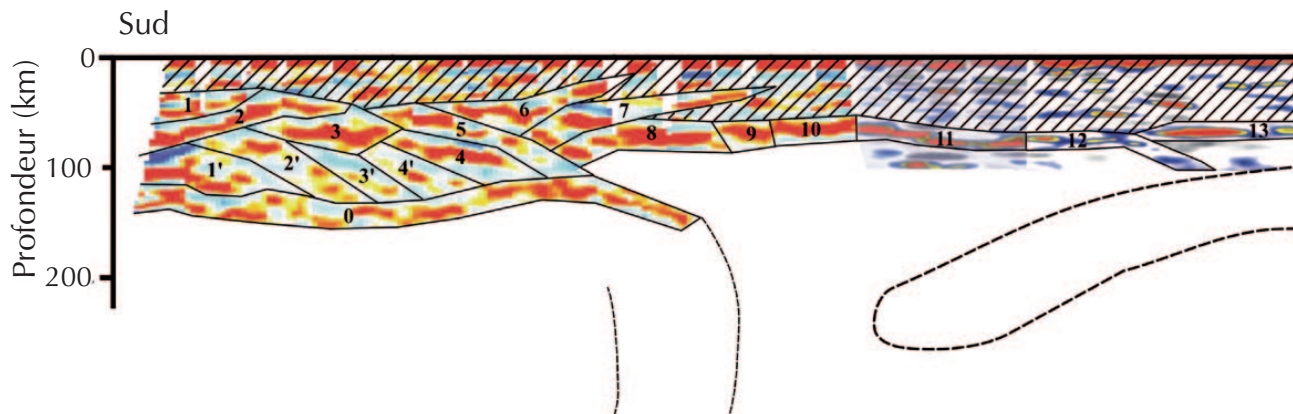

1. Coupe Sud-Nord du Tibet, obtenue à partir des campagnes sismologiques de I'INSU entre 1981 et 1998 (A. Hirn). La base de la croûte (zone hachurée, discontinuité de Mohorovičić, entre 50 et 70 km) n'est pas plane et semble souvent multiple. Des interfaces plus profondes apparaissent aussi.

\section{Neutrinos et problèmes inverses}

L'annonce faite le 22 septembre 2011 par les chercheurs de l'expérience OPERA [1], que la vitesse des neutrinos observés dépassait celle de la lumière, a fait l'effet d'une bombe, non seulement dans les milieux scientifiques, mais dans l'ensemble de la presse. Notre propos ici n'est pas de faire l'analyse d'une expérience fort complexe, ou de discuter la validité de ses résultats, mais de montrer qu'une théorie très générale y est discrètement à l'œuvre, comme dans des dizaines d'applications faites par chacun de nous dans la vie de tous les jours.

Rappelons d'abord que l'expérience est basée sur la création de neutrinos au CERN près de Genève et la détection de leur arrivée à une station, sous le Gran Sasso dans les Abruzzes. Les neutrinos font le parcours en ligne droite dans la croûte terrestre. La longueur du trajet parcouru est de l'ordre de $730 \mathrm{~km}$. Pour la déterminer, on a procédé par GPS à des mesures d'une extrême précision [2].

Or, localisation par GPS, ingénierie médicale (échographie, tomographie...), recherche de défauts par analyse non destructive, reconnaissance de structures et de caractères, détection de nouvelles planètes, structure de l'intérieur de la Terre (fig. 1) et d'étoiles, recherche pétrolière ou gazière, et bien d'autres applications - de la diffraction de particules en mécanique quantique [3] à l'économétrie -, ne seraient pas possibles sans les progrès faits pendant ces cinquante dernières années dans la théorie des problèmes inverses (et sans l'explosion de la puissance des ordinateurs nécessaires pour leur résolution).

Dans le cas du GPS, il a fallu d'abord résoudre le problème inverse de la détermination du champ de gravité de la Terre à partir de l'observation des trajectoires de nombreux satellites (la Lune d'abord, puis les satellites artificiels). Une fois ce champ connu, les positions d'un groupe de satellites munis d'émetteurs et d'horloges très précises peuvent être déterminées. La position d'un point à la surface de la Terre peut alors être calculée à partir des temps d'arrivée des ondes émises par les satellites. En fait, l'amélioration des modèles du champ de gravité se fait en traitant simultanément les deux problèmes : calcul des trajectoires et positionnement des stations. C'est un des aspects particulièrement intéressants de la théorie d'Albert Tarantola (voir sa biographie, encadré 1, p. 14), présentée in fine. 


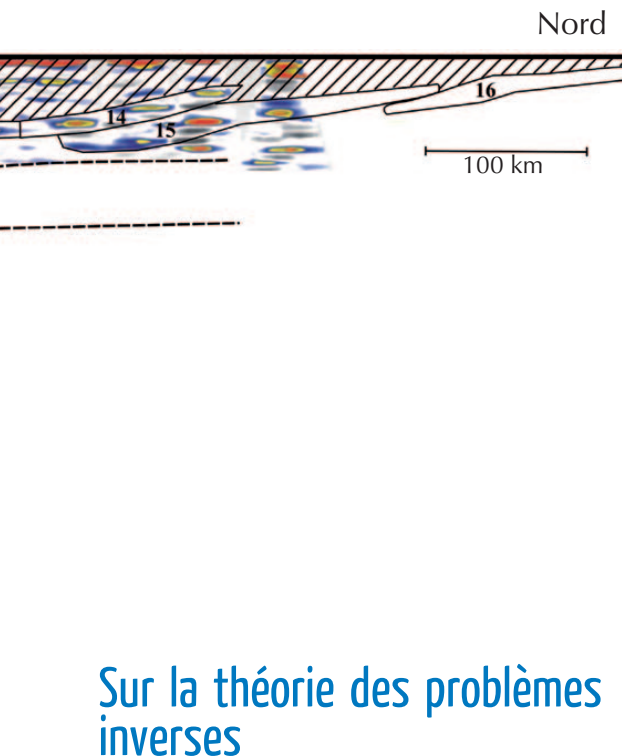

\section{Sur la theorie des problemes}

Je vais essayer de donner une idée des méthodes de la théorie des problèmes inverses, en évitant, autant que possible, les aspects techniques.

On peut décrire ainsi la démarche habituelle d'une science expérimentale : les phénomènes observés conduisent à formuler des hypothèses et à élaborer une théorie, dans laquelle on construit un modèle du système étudié, prenant en compte les paramètres nécessaires à sa caractérisation. Cette théorie permet alors de résoudre le problème dit " direct ", c'est-à-dire de calculer, à partir des valeurs choisies pour les paramètres pertinents, les valeurs de certaines grandeurs, les données, que l'on comparera aux valeurs observées. Une théorie est en somme la formulation d'un problème direct. Prenons comme exemple un problème étudié par Abel en 1826 : sur une ligne de plus grande pente d'une colline (supposée de forme connue, et parfaitement lisse) on lance un mobile vers le haut. Connaissant le profil de la colline et la vitesse initiale du véhicule, trouver la durée d'un trajet aller-retour. En comparant les valeurs calculées aux valeurs observées, éventuellement à la suite d'expériences

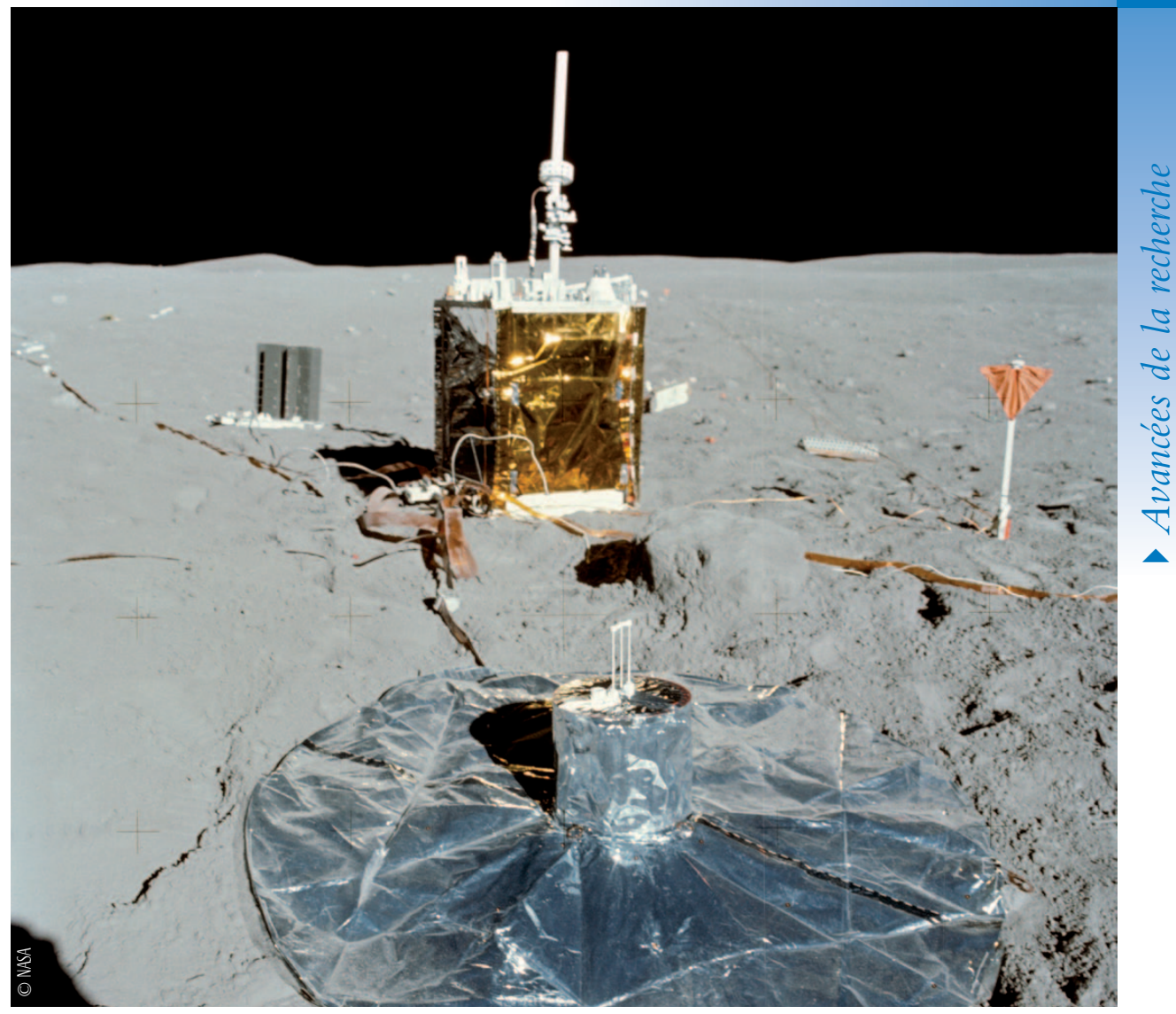

Le sismomètre passif déposé sur la Lune lors de la mission Apollo 16 en avril 1972 (en bas, au centre).

programmées à cette fin, on peut vérifier la validité de la théorie ou la réfuter.

Déterminer les propriétés du système étudié à partir des données observées, c'est-à-dire résoudre un problème inverse, est une démarche autrement difficile, car elle procède à l'opposé de la démarche normale des sciences expérimentales. Pour le cas étudié par Abel, le problème inverse consiste à trouver la forme du profil de la colline, connaissant le temps mis pour un aller-retour du mobile en fonction de sa vitesse initiale. En mathématiques, c'est par exemple le fameux problème du tambour : trouver sa forme à partir des sons qu'on en tire ; il faut alors chercher les coefficients d'une équation aux dérivées partielles ou d'une équation différentielle, connaissant le spectre des valeurs propres. On rencontre ce type de problème, en particulier quand on veut déterminer les propriétés d'un milieu inaccessible à l'observation directe, à partir d'observations faites à l'extérieur de celui-ci.

C'est évidemment le cas de la géophysique interne. Il est donc naturel que de nombreux géophysiciens aient contribué aux progrès de cette discipline en développant toute une série de méthodes pour résoudre leurs problèmes, qu'ils soient linéaires, linéarisables ou non.

\section{Problèmes inverses en géophysique}

Un des premiers problèmes inverses étudiés dans cette discipline est la détermination de l'origine (position, instant) des ondes émises lors d'un séisme, à partir de leurs temps d'arrivée en un certain nombre de points. Si l'on peut supposer que la propagation se fait à vitesse constante sur une surface plane, ce problème n'offre pas de difficulté et peut être traité par la méthode des moindres carrés développée par Gauss au début du $\mathrm{XIX}^{\mathrm{e}}$ siècle. Une fois déterminée la source, on peut calculer le temps mis par une onde pour atteindre un point donné de la surface. On constate que sur la Terre (presque) sphérique, cette durée ne dépend (presque) que de la distance entre la source et la station, et que la vitesse apparente du signal est plus grande que si la propagation se faisait en surface.

On peut alors formuler un autre problème inverse : déterminer la vitesse de l'onde en fonction de la profondeur, si elle n'est fonction que de celle-ci. Vers 1910, Batemann, Herglotz et Wiechert appliquèrent la méthode employée par Abel au problème cité plus haut qui lui est équivalent. Dans la pratique, on rencontre cependant beaucoup de difficultés. La solution 


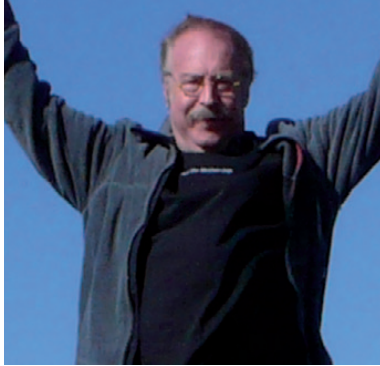

Albert Tarantola a fait toute sa carrière à l'Institut de Physique du Globe de Paris. Mais il s'était d'abord intéressé à l'astronomie, avec pour thèse de spécialité : Étude en relativité générale de l'évolution des amas de galaxies. L'enseignement suivi laissa en lui une marque profonde et orienta nombre de ses réflexions.

Il choisit ensuite la géophysique interne et s'intéressa aussitôt à la théorie des problèmes inverses que j'enseignais alors. Il eut très vite I'intuition qu'une approche probabiliste permettrait une meilleure compréhension de la question. En 1987, il publia chez Elsevier le livre Inverse Problem Theory [1], dans lequel il développe les idées présentées dans sa thèse. A. Tarantola sut fonder une excellente école de géophysique appliquée. II en fut un spécialiste reconnu dans le monde entier, et ses travaux furent les plus cités en géophysique dans la seconde moitié du XXe siècle.

Mais ses études initiales en relativité l'amenèrent à une nouvelle entreprise, qu'il considérait comme une véritable refonte de la physique. Pour lui, effectuer une mesure ne consiste pas simplement à obtenir la valeur d'une grandeur physique avec l'incertitude qui lui est attachée. Car l'objet étudié est en fait une « qualité » du système, qui peut correspondre à diverses grandeurs selon le phénomène. Par exemple, pour la qualité chaud-froid d'un système donné, on pourra utiliser la température ou son inverse, ou son exponentielle pondérée. En musique, la fréquence ou la période... Ces outils ne sont en fait que les coordonnées que l'on a choisies sur une variété abstraite, qu'il appelle l'espace de la " qualité mesurable ». En étudiant la géométrie de la variété, on pourra déduire une théorie invariante par rapport à ce choix. On élimine de la sorte l'anomalie observée, par exemple en sismologie dans la résolution d'un problème inverse, où le résultat obtenu change selon que l'on cherche à déterminer la vitesse ou son inverse, la lenteur. Mais cette approche obligea A. Tarantola à construire un véritable arsenal mathématique, car la torsion de la variété fait perdre leur caractère associatif aux lois des ensembles étudiés. Ces notions sont développées dans son ouvrage Elements for Physics [2], où il présente aussi une nouvelle approche de la théorie de l'élasticité.

A. Tarantola pensait être en mesure de faire progresser significativement la théorie des probabilités. Il considérait que la théorie de Kolmogoroff, basée sur les probabilités conditionnelles, était insuffisante pour traiter le cas réel où les données sont entachées d'erreurs. Il préparait un ouvrage, Mapping of probabilities, qu'il ne put achever.

Albert Tarantola avait une personnalité généreuse, attachante, à l'ego flamboyant, mais surtout très originale. Né à Barcelone, il a passé toute sa jeunesse en Espagne, avant d'arriver à Paris au début des années 1970. Il abandonna très jeune le collège pour travailler, et ne reprit ses études que beaucoup plus tard, rattrapant rapidement son retard. Ce cursus hétérodoxe explique peut-être en partie son approche très personnelle de tous les problèmes qu'il a abordés : il combinait sa vive intelligence rationnelle et son ignorance

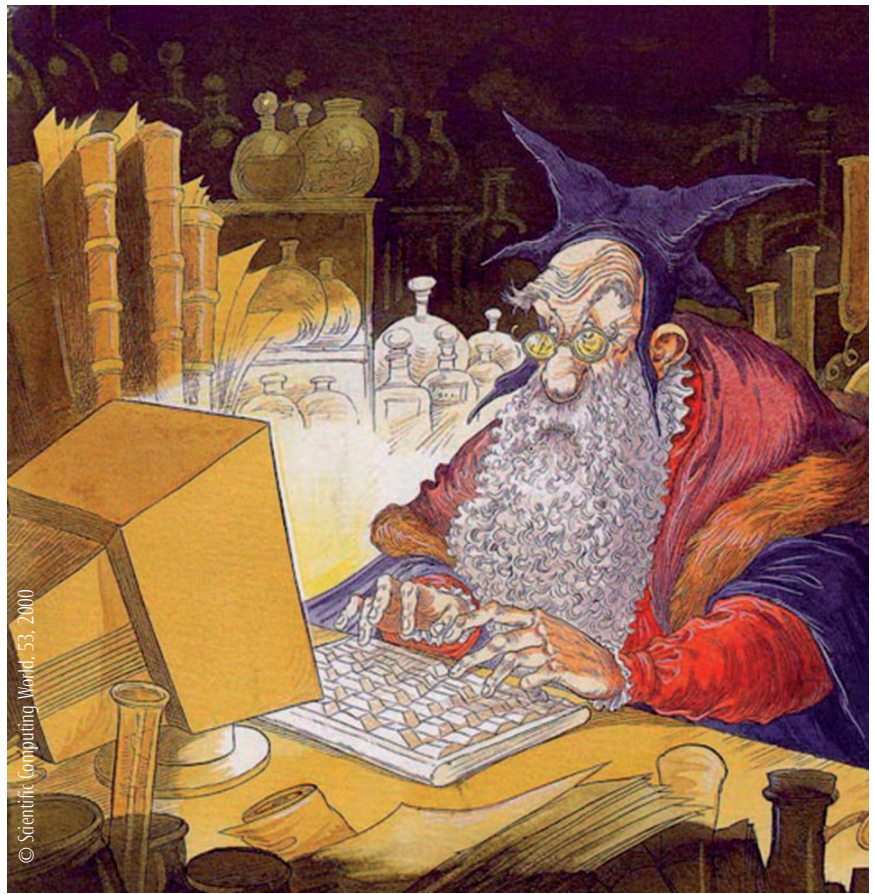

Dessin représentant Albert Tarantola en alchimiste dans son laboratoire. (Source : site web personnel d'Albert Tarantola)

initiale des outils nécessaires, qu'il reconstruisait à sa manière. Cet aspect, bien visible dans son dernier ouvrage, est sans doute une des raisons qui n'ont pas permis que son travail fût mieux connu et discuté. Seul le premier des chantiers qu'il avait lancés, a pu être mené à bien. Mais peut-être que les idées qu'il a présentées dans les autres, trouveront un jour leur place en physique.

Georges Jobert

[1] A. Tarantola, Inverse problem theory - Methods for data fitting and model parameter estimation, Elsevier Science and Technology (1987).

\section{[2] A. Tarantola, Elements for Physics - Quantities, Qualities, and Intrinsic Theories, Springer (2006).}

- Site Internet personnel d'Albert Tarantola :

www.ipgp.fr/ tarantola/
- on peut y télécharger les fichiers pdf de ses deux livres et de ses
principaux articles,
- accéder par un lien à ses notes de cours
(www.ipgp.fr/ tarantola/Files/Professional/Teaching/index.html),
- et y découvrir ses opinions - son désaccord avec l'expérimentation
animale et sa technophobie, en particulier.




\section{\>}

nécessite en effet le calcul de la dérivée du temps de transit par rapport à la distance, opération peu fiable avec des résultats expérimentaux. De plus, comme l'ont montré des géophysiciens soviétiques dans les années 1960, elle n'est unique que si la loi de vitesse satisfait certaines conditions. Mais cette méthode a permis de montrer que, dans l'ensemble, la vitesse croît avec la profondeur, avec, à certaines profondeurs des discontinuités, comme celle mise en évidence par le sismologue serbe Mohorovičić, en 1909, à une trentaine de $\mathrm{km}$ de profondeur.

Gutenberg montra l'existence du noyau terrestre en 1913. Des modèles de plus en plus détaillés de l'intérieur du Globe furent ensuite obtenus. En 1967, deux géophysiciens américains, G. Backus et F. Gilbert, développèrent une théorie générale qui amena une véritable révolution dans la discipline (voir la section « Les progrès » et la référence [4]).

\section{Approximations successives}

Les propriétés d'un milieu dépendent en principe de la position et du temps. Une description complète nécessiterait donc en général une information infinie. Dans la pratique, en se limitant à des problèmes statiques et en fragmentant le milieu, on se ramène à des suites discrètes et finies de paramètres. Par exemple, en définissant un modèle de Terre par la valeur de la densité dans une décomposition en volumes élémentaires homogènes, on peut, par la théorie de la gravitation de Newton, en déduire l'attraction qu'elle exerce à son extérieur.

On peut procéder ensuite, par ajustements successifs des paramètres, à l'obtention d'un accord entre valeurs calculées et valeurs observées, compatible avec la précision instrumentale. C'est l'approche classique par approximations successives, la seule appliquée jusqu'au milieu du siècle dernier.

\section{Existence, unicité, stabilité des solutions pour un problème linéaire}

Les problèmes linéaires - c'est-à-dire ceux où les données dépendent linéairement des paramètres - sont les seuls pour lesquels la théorie peut être développée complètement (encadré 2). Un exemple simple permet de se rendre compte des difficultés qu'on y rencontre.

Supposons que les données $\mathrm{d}_{1}$ et $\mathrm{d}_{2}$ dépendent linéairement des paramètres $\mathrm{x}_{1}$ et $\mathrm{x}_{2}$, suivant le système :

$\mathrm{x}_{1}+\mathrm{x}_{2}=2 \mathrm{~d}_{1}, \mathrm{x}_{1}+\mathrm{x}_{2}=2 \mathrm{~d}_{2}$.

Ce système n'a pas de solution si $\mathrm{d}_{1} \neq \mathrm{d}_{2}$; mais si $d_{1}=d_{2}$, il en a une infinité : $\mathrm{x}_{1}=\mathrm{d}_{1}+\mathrm{a}, \mathrm{x}_{2}=\mathrm{d}_{1}-\mathrm{a}$, où a est quelconque. De plus, la valeur des solutions peut dépendre très fortement de celle des coefficients de l'équation. Par exemple, si la seconde équation du système était remplacée par: $\mathrm{x}_{1}+1,001 \mathrm{x}_{2}=2 \mathrm{~d}_{2}$,

le système aurait alors une solution unique :

$$
\begin{gathered}
\mathrm{x}_{1}=2 \mathrm{~d}_{1}-2000\left(\mathrm{~d}_{2}-\mathrm{d}_{1}\right) \\
\mathrm{x}_{2}=2000\left(\mathrm{~d}_{2}-\mathrm{d}_{1}\right) .
\end{gathered}
$$

Pour $\mathrm{d}_{2}=\mathrm{d}_{1}=1$, on aurait $\mathrm{x}_{1}=2$ et $\mathrm{x}_{2}=0$; mais pour $\mathrm{d}_{1}=1$ et $\mathrm{d}_{2}=1,01$, on aurait $\mathrm{x}_{1}=-18$ et $\mathrm{x}_{2}=20$.

On voit que si les données ou la théorie ne sont connues qu'avec une certaine précision, la solution peut varier énormément pour une petite variation des données; le système est instable, et le problème est dit " mal posé ". La matrice qui fait passer des paramètres aux données, est alors qualifiée de «mal conditionnée ».

\section{Les progrès}

On ne doit pas chercher une solution dépendant d'un nombre de paramètres supérieur au nombre de données. C'est une forme du principe de parcimonie (ou rasoir d'Ockham). Mais toutes les données n'ont pas la même importance. On peut tenir compte de l'information dont on dispose sur leurs erreurs probables, en donnant plus de poids à celles qui sont les plus précises. On introduit pour ce faire des matrices dites de covariance des données et de covariance des paramètres. On peut ainsi construire un inverse dit généralisé, qui est, dans un certain sens, la meilleure solution accessible.

G. Backus et F. Gilbert ont montré [4] en particulier qu'on peut, avant toute mesure effective, évaluer le nombre d'informations indépendantes contenues dans une collection de données - ce qu'on appelle la distribution de l'information. On peut, en principe, déterminer, avant toute expérience, quelles sont celles qui fourniront les données les plus intéressantes. On peut parallèlement évaluer le pouvoir séparateur qu'on peut alors obtenir sur les valeurs des paramètres du modèle. Une valeur locale pour un paramètre pouvant être considérée comme une moyenne pondérée par un certain filtre, le pouvoir séparateur correspond à la largeur du filtre optimal, qui peut être évaluée à partir des données.

Avec des données expérimentales, les résultats ont une imprécision due à la fois aux erreurs sur ces données et au pouvoir séparateur fini. On peut chercher à minimiser une quantité qui combine ces effets. Pour les problèmes linéaires, on montre que lorsque l'on fait décroître l'erreur expérimentale en augmentant la précision

\section{Problèmes linéaires : un peu de maths}

encadré 2

Si l'on considère la collection de données comme un vecteur $\mathrm{D}$ et celle des paramètres comme un vecteur $\mathrm{M}$, la relation linéaire du problème direct peut s'écrire sous la forme : $D$ = GM, où G est une matrice. La transformation linéaire de $M$ vers $D$ est la composition d’homothéties suivant les axes principaux de la transformation, ses « vecteurs propres », avec pour facteurs leurs " valeurs propres ». Le domaine de l'espace des paramètres sous-tendu par les vecteurs propres à valeurs propres non nulles, est transformé par G dans l'espace des données en ce qu'on appelle l'image de M. Le système n'a de solution que si D est dans cette image. Le domaine complémentaire, sous-tendu par les vecteurs propres à valeurs propres nulles, s'appelle le noyau de G. Pour des paramètres appartenant au noyau, les données correspondantes sont nulles. On peut donc obtenir une nouvelle solution, en ajoutant à une première solution tout vecteur du noyau. II n'y a pas unicité.

$\mathrm{L}^{\prime}$ opération inverse $\mathrm{G}^{-1}$, qui fait passer de $\mathrm{D}$ à $\mathrm{M}$, se fait en composant des homothéties suivant les mêmes directions, mais avec des valeurs propres inverses des précédentes. (Dans l'exemple numérique ci-dessus, une valeur propre est voisine de 2, I'autre de 0,001. La matrice inverse a des éléments de l'ordre de 2000). En l'absence de valeurs propres nulles, cet inverse existe, et le problème inverse n'a qu'une solution : $M=G^{-1} \mathrm{D}$. Mais comme on I'a vu, $\mathrm{G}$ peut être mal conditionnée : l'erreur relative sur la solution produite par une variation relative des données peut être un très grand multiple de cette dernière. 
des mesures, le pouvoir séparateur décroit. Inversement, chercher une meilleure localisation pour un paramètre conduit à augmenter l'erreur que l'on a sur lui. C'est une forme du principe d'incertitude.

\section{Problèmes non linéaires}

Les problèmes non linéaires ne permettent pas ce genre d'analyse. Pour un modèle donné, on peut évaluer l'écart entre données observées et données calculées - en calculant par exemple la somme des carrés des différences, d'où le nom : méthode des moindres carrés, ou celle de leurs modules - et chercher la solution dans la direction où cet écart diminue le plus vite (méthode du gradient). On aboutit cependant souvent de cette manière à des minima secondaires, qui ne donnent pas la valeur la plus faible. Deux autres méthodes peuvent être utilisées :

a) Linéarisation. Si les études antérieures fournissent un résultat valable en première approximation, on peut chercher une solution au voisinage de ce modèle. Notant : $D=G(M)$ la relation entre données et modèle, $\mathrm{M}_{0}$ le modèle de départ, $\varepsilon$ son écart à la solution cherchée, on écrira : $\mathrm{D}=\mathrm{G}\left(\mathrm{M}_{0}+\varepsilon\right)=\mathrm{G}\left(\mathrm{M}_{0}\right)+\varepsilon \mathrm{G}^{\prime}\left(\mathrm{M}_{0}\right)+\mathrm{O}\left(\varepsilon^{2}\right)$, où $G$ ' est une fonctionnelle que l'on peut construire à partir de G. On cherchera alors la solution du problème linéaire :
$\mathrm{D}=\mathrm{G}\left(\mathrm{M}_{0}\right)+\varepsilon \mathrm{G}^{\prime}\left(\mathrm{M}_{0}\right)$. Ce processus peut être itéré.

b) Méthode de Monte-Carlo. Si le coût en calcul de la solution du problème direct n'est pas excessif, on peut procéder à une exploration aléatoire du domaine des paramètres. On évite ainsi la difficulté mentionnée plus haut. Mais dès que le nombre de paramètres est un peu grand, cette recherche devient vite trop coûteuse.

On trouvera une présentation détaillée de ces diverses méthodes dans les ouvrages de A. Tarantola [5]. Ce dernier a proposé [6] une approche probabiliste, basée sur un postulat dû à Bayes (1702-1761). L'encadré 3 en donne un aperçu très sommaire.

Dans cette démarche, problème direct et problème inverse perdent leurs spécificités et sont en fait remplacés par la recherche de l'état d'un système. Ceci correspond aussi à l'évolution de la recherche : par exemple, en sismologie on ne cherche plus à déterminer séparément les paramètres de la source sismique (origine et mécanisme au foyer) et ceux de l'intérieur du Globe. On cherche à déterminer simultanément tous ces paramètres, en tirant parti non seulement des temps d'arrivée des ondes, mais aussi de la forme des sismogrammes.

On peut adopter la formulation d'A. Tarantola : on dispose d'une information a priori sur les paramètres du modèle étudié, sous la forme d'une distribution de probabilités sur l'espace des modèles. Cette distribution est transformée en une distribution a posteriori, en incorporant d'une part, une théorie physique qui relie les paramètres aux grandeurs observables, et qui peut éventuellement être elle-même entachée d'erreurs - et, d'autre part, une information fournie par les données observées. On effectue ainsi une révision de notre connaissance de l'état du système.

\section{Références}

1• F. Vannucci, « Les neutrinos défient la relativité », Reflets de la Physique, 27 (décembre 2011), pp.18-19.

2- Des corrections relativistes sont alors nécessaires. Voir : A. Tarentola et al., "Gravimetry, Relativity, and the Global Navigation Satellite Systems", arXiv:0905.3798v3 (mai 2009)

3• K. Chadan et P.C. Sabatier, Inverse Problems in Quantum Scattering Theory, Springer-Verlag (1977).

4• G. Backus et F. Gilbert, Geophys. J. Roy. Astron. Soc., 13 (1967) 247-276.

5- A. Tarantola, Inverse problem theory and methods for model parameter estimation, SIAM (2005). Tarantola y insiste sur la nécessité de rendre les résultats insensibles au choix de la paramétrisation. On doit pouvoir traiter de façon identique la recherche de la vitesse ou celle de la lenteur des ondes, de la masse ou du volume spécifiques....

6• A. Tarantola et B. Valette, "Inverse Problems = Quest for Information”, J. Geophys., 50 (1982) 159-170.

\section{L'approche probabiliste d'Albert Tarantola}

On dispose au départ d'une loi de probabilité $\rho_{\mathrm{D}}(\mathbf{d})$ sur les observables $\mathbf{d}$ et de l'information a priori sur le modèle $\mathbf{m}$ avant d'appliquer le processus d'inversion (par exemple : paramètres compris entre certaines valeurs), représentée par une loi de probabilité $\rho_{M}(\mathbf{m})$. Données et paramètres étant indépendants, l'information a priori qu'on a sur le système est représentée par la loi produit : $\rho(\mathbf{d}, \mathbf{m})=\rho_{D}(\mathbf{d}) \rho_{M}(\mathbf{m})$.

Dans le problème direct, on dispose d'une théorie qui donne $\mathbf{d}$ connaissant $\mathbf{m}$. Cette théorie peut également $\mathbf{n}^{\prime}$ être connue qu'approximativement - à cause des constantes physiques utilisées (toujours déterminées expérimentalement), ou à cause d'erreurs de modélisation (par exemple, modèle sphérique pour la Terre aplatie) - et doit donc aussi être représentée par une loi de probabilité $\Theta(\mathbf{d}, \mathbf{m})$. En combinant ces deux états d'information indépendants, on obtient la probabilité résultante sur les données : $\rho_{D}(\mathbf{d})=\Theta(\mathbf{d}, \mathbf{m}) \cdot \rho_{M}(\mathbf{m})$.

L'observation conduit à la définition de la loi $\sigma_{D}(\mathbf{d})$. La solution du problème inverse est donnée par : $\sigma_{M}(\mathbf{m})=\Theta(\mathbf{d}, \mathbf{m}) . \sigma_{D}(\mathbf{d})$. L'information a posteriori qu'on a sur le système est représentée par la loi produit : $\sigma(\mathbf{d}, \mathbf{m})=\sigma_{\mathrm{D}}(\mathbf{d}) \sigma_{\mathrm{M}}(\mathbf{m})$.
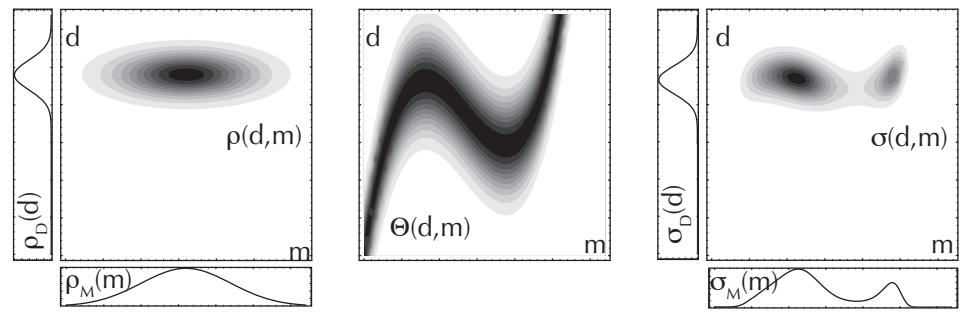

Combinaison des distributions sur les données et les modèles avec celle de la théorie (figure 1.12 de l'ouvrage de Tarantola [5]). Les valeurs de gris correspondent à la valeur du produit.

- À gauche : $\rho_{D}(\mathbf{d})$ et $\rho_{M}(\mathbf{m})$ représentent respectivement l'information sur la donnée $\mathbf{d}$ et celle a priori sur le paramètre $\mathbf{m}$. $\rho(\mathbf{d}, \mathbf{m})$ représente l'information produit.

- Au centre : $\Theta(\mathbf{d}, \mathbf{m})$ représente la relation théorique entre $\mathbf{d}$ et $\mathbf{m}$, avec les erreurs associées.

- À droite : $\sigma(\mathbf{d}, \mathbf{m})$ représente la combinaison des informations a priori et de l'information théorique. $0 \mathrm{n}$ en déduit les probabilités marginales $\sigma_{0}(\mathbf{d})$ et $\sigma_{M}(\mathbf{m})$. On voit en quoi la connaissance du modèle a été améliorée grâce aux données utilisées. Deux types de solutions apparaissent possibles. 